PROCEEDINGS OF THE

AMERICAN MATHEMATICAL SOCIETY

Volume 126, Number 11, November 1998, Pages 3279-3282

S 0002-9939(98)04511-0

\title{
MACKEY WEAK BARRELLEDNESS
}

\author{
STEPHEN A. SAXON AND L. M. SÁNCHEZ RUIZ \\ (Communicated by Dale Alspach)
}

\begin{abstract}
Answers to questions of Levin and Saxon (1971) and of Ferrando and López Pellicer (1991) complete a linear picture of weak barrelledness for Mackey spaces.
\end{abstract}

\section{The Mackey PICture}

A space $E$ (Hausdorff, locally convex with real or complex scalar field $\mathbb{K}$ ) is inductive (cf. [9]; in [3], weakly barrelled) if, given any seminorm $p$ and an increasing covering sequence $\left\{E_{n}\right\}_{n}$ of subspaces such that each $\left.p\right|_{E_{n}}$ is continuous, $p$ itself is continuous. Primitive spaces (cf. $[9,12])$ are defined by replacing the seminorm $p$ by linear form $f$. Obviously, inductive $\Rightarrow$ primitive. But if $E$ is primitive, its dual agrees with that of the inductive limit of any increasing covering sequence of subspaces, so that $E$, if Mackey, is inductive. That is, inductivity and primitivity, which comprise box 4) of [9], coincide in the class of Mackey spaces.

A space $E$ is dual locally complete (dlc) if $\left(E^{\prime}, \sigma\left(E^{\prime}, E\right)\right)$ is locally complete. Clearly, dlc $\Rightarrow$ primitive via Ruess' property (LC) (cf. [5, 8.1.29(i)], [8], [13]).

Precise knowledge of which conditions coincide for Mackey spaces facilitates "Reinventing weak barrelledness" [9], whose general two-dimensional picture gathers $C$-barrelled, $c_{0}$-barrelled, property (L) and dlc into box 2). Section 3 answers the Ferrando-López Pellicer question [2] to show that the four notions of box 2) coincide for Mackey spaces. The eleven distinct weak barrelledness conditions (cf. $[4,5,9])$ then become just seven linearly related ones:

$$
\begin{gathered}
\text { Under the Mackey Topology } \\
\text { barrelled } \Rightarrow \aleph_{0} \text {-barrelled } \Rightarrow \ell^{\infty} \text {-barrelled } \\
\Rightarrow \text { property }(\mathrm{C}) \Rightarrow \text { property }(\mathrm{S}) \Rightarrow \text { box } 2) \Rightarrow \text { box } 4) .
\end{gathered}
$$

The examples of $[3,10]$ show that, even for metrizable spaces, we cannot reverse the last arrow. Section 2 answers the Levin-Saxon question to show that the third arrow is irreversible, as are the remaining four via [4, 5, 13], so that the above scheme tells the entire Mackey space relational story.

Received by the editors March 20, 1997.

1991 Mathematics Subject Classification. Primary 46A08.

Key words and phrases. Weak barrelledness, Mackey topology, Levin-Saxon and FerrandoLópez Pellicer questions.

Support from the Consellería de Educación y Ciencia en el marco del Plan Valenciano de Ciencia y Tecnología, also from DGICYT PB94-0535 and IVEI 033/003, is gratefully acknowledged. 


\section{TWO EXAMPLES FOR LEVIN-SAXON}

Recall $[4,5]$ that a space $E$ is $\ell^{\infty}$-barrelled, respectively, has property $(C)$, if every $\sigma\left(E^{\prime}, E\right)$-bounded sequence $\left\{f_{n}\right\}_{n}$ is equicontinuous, respectively, has a weak adherence point $f \in E^{\prime}$; i.e., each $\sigma\left(E^{\prime}, E\right)$-neighborhood of $f$ contains $f_{n}$ for infinitely many $n$. Levin and Saxon wondered [4, p.102] whether a Mackey space with property (C) must be $\ell^{\infty}$-barrelled. Ferrando and Sánchez Ruiz [3] gave a positive answer for the separable case, but we show the general answer is negative.

$\mathbb{K}^{\mathbb{K}}$ denotes the vector space of all functions from $\mathbb{K}$ into $\mathbb{K}$, and $\mathbb{K}^{(\mathbb{K})}$ those functions with finite support. Now $E=\mathbb{K}^{(\mathbb{K})}$ is $\mathfrak{c}$-dimensional and the algebraic dual $E^{*}$ is identified with $\mathbb{K}^{\mathbb{K}}$ in the usual manner, so that the $\sigma\left(E^{*}, E\right)$ topology corresponds to the product topology on $\mathbb{K}^{\mathbb{K}}$. If $I=\{x \in \mathbb{K}:|x| \leq 1\}$, then, because separability is c-multiplicative [1, p.111], there is a sequence $S$ dense in $I^{\mathbb{K}}$, and $S$ is clearly $\sigma\left(E^{*}, E\right)$-bounded. The first uncountable ordinal $\omega_{1}$ is the set of all smaller ordinals and has cardinality $\aleph_{1}$. Let $\mathcal{F}$ be the family of all $\mathfrak{c}$-dimensional subspaces of $E^{*}$ containing $S$, and for each ordinal $\alpha \leq \omega_{1}$, let $\mathcal{F}_{\alpha}$ be the set of all maps $T$ from $\alpha$ into $\mathcal{F}$ such that $\gamma<\beta<\alpha$ implies $T(\beta)$ contains a $\sigma\left(E^{*}, E\right)$-adherence point of each $\sigma\left(E^{*}, E\right)$-bounded sequence in $T(\gamma)$; necessarily, $T(\beta)$ contains $T(\gamma)$. While $\mathcal{F}_{0}$ is trivial, $\mathcal{F}_{1}$ is the set of maps from $1=\{0\}$ into $\mathcal{F}$. We define a partial order $\preceq$ on $\bigcup_{\alpha \leq \omega_{1}} \mathcal{F}_{\alpha}$ by writing $T \preceq U$ to mean that $T \in \mathcal{F}_{\alpha}$ and $U \in \mathcal{F}_{\beta}$ for some $\alpha \leq \beta$ with $T=\left.U\right|_{\alpha}$. Easily, each chain has an upper bound, and Zorn's Lemma provides a maximal element $M$ contained in some $\mathcal{F}_{\mathfrak{m}}$.

Example 2.1. The subspace $E^{\prime}=\bigcup_{\beta \in \mathfrak{m}} M(\beta)$ of $E^{*}$ separates points of $E$, and the Mackey space $\left(E, \mu\left(E, E^{\prime}\right)\right)$ has property $(\mathrm{C})$ but is not $\ell^{\infty}$-barrelled.

Proof. Obviously, $\mathfrak{m} \geq 1$ and $E^{\prime} \in \mathcal{F}$. To choose the aggregate set $A \subset E^{*}$ we select a $\sigma\left(E^{*}, E\right)$-adherence point for each $\sigma\left(E^{\prime}, E\right)$-bounded sequence (Tychonoff's Theorem). The number of such sequences is $\left|E^{\prime}\right|^{\aleph_{0}}=2^{\aleph_{0} \cdot \aleph_{0}}=\mathfrak{c}$, and thus $|A|=\mathfrak{c}$. Now $\operatorname{sp}(A) \in \mathcal{F}$, and we may define $W: \mathfrak{m}+1 \rightarrow \mathcal{F}$ by writing $W(\mathfrak{m})=\operatorname{sp}(A)$ and $\left.W\right|_{\mathfrak{m}}=M$. It is apparent that $\mathfrak{m}=\omega_{1}$, for otherwise, $\mathfrak{m}+1 \leq \omega_{1}$ implies $\mathcal{F}_{\mathfrak{m}+1}$ exists, and $W \in \mathcal{F}_{\mathfrak{m}+1}$ contradicts the maximality of $M$.

Since the set $I^{\mathbb{K}}$ separates points of $E$, so does the weakly dense subset $S$, and hence so does $E^{\prime}$. Now suppose $R$ is any $\sigma\left(E^{\prime}, E\right)$-bounded sequence. As the cofinality of $\mathfrak{m}=\omega_{1}$ is uncountable, there exists $\beta \in \mathfrak{m}$ such that $R \subset M(\beta)$, which implies that there is a weak adherence point of $R$ in $M(\beta+1) \subset E^{\prime}$; i.e., the Mackey space $E$ has property $(\mathrm{C})$.

But $E$ cannot be $\ell^{\infty}$-barrelled, for if $S$ were equicontinuous, then its $\sigma\left(E^{*}, E\right)$ closure $I^{\mathbb{K}}$ would be contained in $E^{\prime}$, forcing $\operatorname{dim}\left(E^{\prime}\right)=2^{\mathfrak{c}} \neq \mathfrak{c}$.

Example 2.2. With $E$ and $E^{\prime}$ as in the previous example, there is a subspace $H$ of $E^{*}$ with $E^{\prime} \subset H$ such that $\operatorname{dim}\left(H / E^{\prime}\right)=\aleph_{0}$, and such that the Mackey space $(E, \mu(E, H))$ has property $(\mathrm{C})$ but is not $\ell^{\infty}$-barrelled.

Proof. $F=\left(E, \sigma\left(E, E^{\prime}\right)\right)$ also has the duality invariant property $(\mathrm{C})$, and has a base of $\mathfrak{c}$ neighborhoods of 0 . Therefore $\operatorname{sub}(F) \leq \operatorname{dom}(F) \leq \mathfrak{c}^{\aleph_{0}}=\mathfrak{c}=\operatorname{dim}(F)$, so that by the proof of the main result in either [7] or [6], there exists $E^{\prime} \subset H \subset E^{*}$, with $\operatorname{dim}\left(H / E^{\prime}\right)=\aleph_{0}$, satisfying the Tweddle-Yeomans Criterion. Hence $\sigma(E, H)$ preserves property $(\mathrm{C})$ via a generalization [11] of the Tweddle-Catalán approach [14]. Duality invariance ensures property (C) for $(E, \mu(E, H))$.

Again, smallness of $\operatorname{dim}(H)=\mathfrak{c}+\aleph_{0}=\mathfrak{c}$ denies $\ell^{\infty}$-barrelledness. 


\section{A theorem for Ferrando-López Pellicer}

The closed unit ball $B$ of the Banach space $\ell^{1}$ is closed in the product $I \times I \times \cdots$, which is compact (Tychonoff); thus we endow $B$ with the topology of coordinatewise convergence to make it compact. We showed [8] that a space $E$ is locally complete if and only if, given any bounded sequence $\left\{x_{n}\right\}_{n} \subset E$ and $\left(a_{n}\right)_{n} \in B$, the series $\sum_{n} a_{n} x_{n}$ converges in $E$. Since null sequences are bounded, such spaces satisfy the convergence hypothesis below.

Lemma 3.1. Let $\left\{C_{n}\right\}_{n}$ be a sequence of nonempty compact absolutely convex subsets of the space $E$ with the property that, given any neighborhood $V$ of the origin, there exists some positive integer $p$ such that $C_{n} \subset V$ for all $n \geq p$. If each series in the the set $C=\left\{\sum_{n} \lambda_{n} x_{n}:\left(\lambda_{n}\right)_{n} \in B\right.$, each $\left.x_{n} \in C_{n}\right\}$ converges in $E$, then $C$ is compact and coincides with the closed absolutely convex hull $\bar{\Gamma}\left(\bigcup_{n} C_{n}\right)$ of the union.

Proof. By Tychonoff's theorem, the product $P=B \times C_{1} \times C_{2} \times \cdots$ is compact. If the map $f: P \rightarrow E$ defined by

$$
f\left(\left(\lambda_{n}\right)_{n}, x_{1}, x_{2}, \ldots\right)=\sum_{n} \lambda_{n} x_{n}
$$

is continuous, then $C=f(P)$ would be compact. A routine argument shows $C$ is absolutely convex, and the obvious containments $\bigcup_{n} C_{n} \subset C \subset \bar{\Gamma}\left(\bigcup_{n} C_{n}\right)$ would then imply $C=\bar{\Gamma}\left(\bigcup_{n} C_{n}\right)$. Thus the continuity of $f$ is the issue at hand.

Pick any $x=\left(\left(\lambda_{n}\right)_{n}, x_{1}, x_{2}, \ldots\right) \in P$ and let $V$ be a closed absolutely convex neighborhood of 0 in $E$. By hypothesis, there is a positive integer $p$ such that $C_{n} \subset \frac{1}{6} V$ for $n>p$. Each $C_{n}$ is bounded, so there exists $\epsilon>0$ such that $|\alpha| \leq \epsilon$ implies $\alpha C_{n} \subset \frac{1}{3 p} V$ for $n \leq p$. Let $U$ be the neighborhood of $x$ consisting of all $y=\left(\left(a_{n}\right)_{n}, y_{1}, y_{2}, \ldots\right) \in P$ such that for $1 \leq n \leq p$ we have $\left|a_{n}-\lambda_{n}\right| \leq \epsilon$ and $y_{n}-x_{n} \in \frac{1}{3} V$. Thus $y \in U$ implies that

$$
\begin{aligned}
f(y)-f(x) & =\sum_{n \leq p}\left(a_{n} y_{n}-\lambda_{n} x_{n}\right)+\sum_{n>p}\left(a_{n} y_{n}-\lambda_{n} x_{n}\right) \\
& =\sum_{n \leq p}\left(a_{n}-\lambda_{n}\right) y_{n}+\sum_{n \leq p} \lambda_{n}\left(y_{n}-x_{n}\right)+\sum_{n>p}\left(a_{n} y_{n}-\lambda_{n} x_{n}\right)
\end{aligned}
$$

is a member of $p\left(\frac{1}{3 p} V\right)+\frac{1}{3} V+\frac{1}{6} V-\frac{1}{6} V=V$; i.e., $f$ is continuous.

A space $E$ is $C$-barrelled [5] if $U=\bigcap_{n} U_{n}$ is a neighborhood of 0 in $E$ whenever $\left\{U_{n}\right\}_{n}$ is a sequence of barrels that are neighborhoods of 0 such that each $x \in E$ is in $U_{n}$ for all but finitely many $n$.

Theorem 3.2. Each Mackey dual locally complete space $E$ is $C$-barrelled.

Proof. Using the definition's notation and taking polars with respect to the usual pairing $\left\langle E, E^{\prime}\right\rangle$, we have each $\{x\}^{\circ} \supset U_{n}^{\circ}$ for almost all $n$, so that almost all $C_{n}=U_{n}^{\circ}$ are contained in any given $\sigma\left(E^{\prime}, E\right)$-neighborhood of 0 . Dual local completeness and the lemma ensure that $\bar{\Gamma}\left(\bigcup_{n} C_{n}\right)$ is weakly compact. The Mackey topology and the bipolar theorem imply that $\left(\bar{\Gamma}\left(\bigcup_{n} C_{n}\right)\right)^{\circ}=\left(\bigcup_{n} C_{n}\right)^{\circ}=\bigcap_{n} C_{n}^{\circ}=\bigcap_{n} U_{n}^{\circ \circ}=U$ is a neighborhood of 0 in $E$. 
Corollary 3.3. If $E$ is a Mackey space, the following assertions are equivalent:

1. $E$ is dual locally complete.

2. $E$ is $c_{0}$-barrelled.

3. E has property (L).

4. $E$ is $C$-barrelled.

Proof. It is well-known that, in general, $C$-barrelled $\Rightarrow\left[c_{0}\right.$-barrelled $\wedge$ property $\left.(\mathrm{L})\right]$ and $\left[c_{0}\right.$-barrelled $\vee$ property $\left.(\mathrm{L})\right] \Rightarrow$ dlc $($ cf. $[5,8.2 .23(\mathrm{a}),(\mathrm{b})$ and 8.2 .7$],[8])$.

As a special case we have 5.1.33 and 8.1.29(i) of [5], the equivalence of (1)-(3).

\section{REFERENCES}

1. R. Engelking, General Topology, Warszawa, PWN 1977. MR 58:18316b; MR 84k:54035

2. J. C. Ferrando and M. López Pellicer, Una propiedad de M. Valdivia, espacios de tipo (L) y condiciones débiles de tonelación, Rev. Re. Ac. Ci. Ex. Fís. y Nat. 83(1991), 25-34. MR 91j:46005

3. J. C. Ferrando and L. M. Sánchez Ruiz, On sequential barrelledness, Arch. Math. 57(1991), 597-605. MR 92j:46005

4. M. Levin and S. Saxon, A note on the inheritance of properties of locally convex spaces by subspaces of countable codimension, Proc. Amer. Math. Soc. 29(1971), 97-102. MR 43:6692

5. P. Pérez Carreras and J. Bonet, Barrelled Locally Convex Spaces, Math. Studies 131, North Holland, 1987. MR 88j:46003

6. S. A. Saxon, Metrizable barrelled countable enlargements, preprint.

7. S. A. Saxon and L. M. Sánchez Ruiz, Barrelled countable enlargements and the dominating cardinal, J. Math. Anal. Appl. 203(1996), 677-685. CMP 97:03

8. S. A. Saxon and L. M. Sánchez Ruiz, Dual local completeness, Proc. Amer. Math. Soc. 125 (1997), 1063-1070. MR 97g:46004

9. S. A. Saxon and L. M. Sánchez Ruiz, Reinventing weak barrelledness, preprint.

10. S. A. Saxon and L. M. Sánchez Ruiz, Metrizable weak barrelledness and dimension, preprint.

11. S. A. Saxon, L. M. Sánchez Ruiz, and I. Tweddle, Countably enlarging weak barrelledness, preprint.

12. S. A. Saxon and I. Tweddle, The fit and flat components of barrelled spaces, Bull. Austral. Math. Soc. 51(1995), 521-528. MR 96c:46005

13. S. A. Saxon and I. Tweddle, Mackey $\aleph_{0}$-barrelled spaces, Advances in Math., to appear.

14. I. Tweddle and F. X. Catalán, Countable enlargements and weak barrelledness conditions, in Topological vector spaces, algebras and related areas, (A. Lau and I. Tweddle, Editors) Pitman RNMS 316, Longman, 1994, pp. 56-65. MR 96a:46011

Department of Mathematics, University of Florida, P.O. Box 118105, Gainesville, FLORIDA 32611-8105

E-mail address: saxon@math.ufl.edu

EUiti-Departamento de Matemática Aplicada, Universidad Politécnica de Valencia, E-46022 Valencia, Spain

E-mail address: lmsr@mat.upv.es 